\title{
Filgotinib in Rheumatoid Arthritis: A Profile of Its Use
}

\author{
Esther S. Kim ${ }^{1}$. Susan J. Keam ${ }^{1}$ (1) \\ Accepted: 15 June 2021 / Published online: 25 July 2021 \\ (c) Springer Nature 2021, corrected publication 2021
}

\begin{abstract}
Filgotinib (Jyseleca ${ }^{\circledR}$ ), an oral Janus kinase (JAK) inhibitor, is approved as monotherapy or in combination with methotrexate to treat moderate to severe active rheumatoid arthritis (RA) in adults who have an inadequate response or intolerance to one or more disease-modifying antirheumatic drugs (DMARDs). In phase 3 trials, once-daily filgotinib was generally well tolerated and associated with an improvement in RA signs and symptoms as well as physical function in patients with an inadequate response to ongoing methotrexate, an inadequate response to ongoing conventional synthetic DMARDs plus an inadequate response or intolerance to prior biologic DMARDs, or limited or no prior exposure to methotrexate. In addition, filgotinib was noninferior to adalimumab in terms of low disease activity response rate (DAS28-CRP $\leq 3.2$ ) in patients with an inadequate response to methotrexate. Filgotinib also appeared to inhibit the radiographic progression of joint damage and led to low disease activity or disease remission (DAS28-CRP < 2.6). Filgotinib showed sustained efficacy, and the safety profile of filgotinib longer term was similar to that in the phase 2 and 3 trials.
\end{abstract}

\section{Plain Language Summary}

Rheumatoid arthritis (RA) is a chronic autoimmune inflammatory disease mainly affecting the small joints of the hands and feet. While there is no cure for RA, biologic disease-modifying antirheumatic drugs (DMARDs), which target the inflammatory cytokines (and their receptors) involved in RA, can achieve low disease activity or disease remission. However, these drugs are not always effective or well tolerated and their administration routes (intravenous or subcutaneous) can be a barrier to use. More recently, oral drugs that act on pathways downstream of cytokine receptors have been developed. These drugs, the Janus kinase (JAK) inhibitors, are targeted synthetic DMARDs. Filgotinib (Jyseleca ${ }^{\circledR}$ ), a second-generation JAK inhibitor, improves joint swelling, disease activity, pain, and physical functioning and reduces progression of joint damage in adults with moderate to severe active RA and is generally well tolerated. Like other JAK inhibitors, filgotinib is recommended in treatment guidelines as an effective alternative to biologic DMARDs in adults with moderate to severe active RA who have not responded adequately to or who do not tolerate other DMARDs.

Digital Features for this Adis Drug Q\&A can be found at https:// doi.org/10.6084/m9.figshare.14562627.

\section{What is the Rationale for Using Filgotinib in RA?}

Rheumatoid arthritis (RA) is a chronic autoimmune inflammatory joint disease characterized by synovitis that typically develops symmetrically in small joints of

Susan J. Keam

demail@springer.com

1 Springer Nature, Private Bag 65901, Mairangi Bay, Auckland 0754, New Zealand the hands and feet [1]. Manifestations include joint swelling, tenderness, warmth and a reduced range of motion. Persistent inflammation can destroy joints and tendons over time, eventually leading to joint deformity. RA can also have extra-articular presentations, with inflammation involving the eyes, skin, heart or lungs [1]. As is the case for most chronic diseases, there is no cure for RA, and the disease will not remit spontaneously [2]. The main therapeutic target for patients with RA is clinical remission, and low disease activity is the best possible alternative [2].

Although the initial cause of RA is unknown, various factors have been implicated in its pathophysiology, including a diverse range of inflammatory cytokines [e.g. tumour necrosis factor, interleukin (IL)-1 $\beta$, IL-6, interferon (IFN)- $\gamma$ ] $[3,4]$. The development of agents targeting cytokines and their receptors, referred to as biologics [or biological 


\section{Adis evaluation of filgotinib in RA}

Second-generation JAK inhibitor with preferential inhibition of JAK1 over JAK2, JAK3 and TYK2.

As monotherapy or combination therapy, improves RA signs and symptoms as well as physical function and inhibits the progression of structural joint damage.

Noninferior to adalimumab in terms of achieving low disease activity.

Generally well tolerated.

disease-modifying antirheumatic drugs (bDMARDs) [2]], was a big advancement in the treatment of autoimmune and inflammatory diseases such as RA [4]. However, targeting a single cytokine does not completely block the progression of RA in all patients, and many bDMARDs lose efficacy over time due to immunogenicity. In addition, the intravenous or subcutaneous routes of administration of bDMARDs may be an obstacle for some patients [4].

Several signal transduction pathways have also been implicated in the progression of RA [3]. For example, the Janus kinase/signal transducers and activators of transcription (JAK/STAT) pathway facilitates the signal transduction of several cytokines and molecules implicated in RA, including IL-6 and IFN- $\gamma$ [3]. An increased understanding of cellular pathways downstream of cytokine receptors has led to the development of small orally available molecules, such as those targeting JAKs, that can simultaneously inhibit multiple cytokines involved in RA [4]. JAK inhibitors are classified as targeted synthetic (ts)DMARDs [5].

First-generation JAK inhibitors [e.g. tofacitinib (a JAK1, JAK2 and JAK3 inhibitor) and baricitinib (a JAK1 and JAK2 inhibitor)] have shown effectiveness in improving inflammatory conditions such as RA, but concerns about adverse effects such as cytopenias associated with nonselective pan-JAK blockade have led to the development of secondgeneration JAK inhibitors with selective inhibitory activity for specific JAKs [4]. Upadacitinib, a JAK1 inhibitor, was the first to be approved [1]. Filgotinib (Jyseleca ${ }^{\circledR}$ ) is the most recent second-generation JAK inhibitor to be approved in the EU for the treatment of RA [5, 6].

This article provides an overview of the pharmacological properties of filgotinib and reviews the clinical data relevant to its use in moderate to severe active RA. Discussion of clinical trials evaluating filgotinib for other immune-mediated inflammatory diseases (e.g. ankylosing spondylitis, inflammatory bowel disease, psoriatic arthritis) [7] is beyond the scope of this article.

\section{How Does Filgotinib Work?}

Filgotinib is an adenosine triphosphate-competitive and reversible inhibitor of the JAK family [consisting of JAK1, JAK2, JAK3 and tyrosine kinase 2 (TYK2)] [5, 6]. In biochemical assays, filgotinib inhibited the activity of JAK 1 preferentially ( $\mathrm{IC}_{50}$ of $10,28,810$ and 116 nM for JAK1, JAK2, JAK3 and TYK2, respectively) [6, 8]. In human cellular assays, filgotinib showed preferential inhibition of JAK1/3, JAK1/2 and JAK1/TYK2, with functional selectivity over cytokine receptors that signal via pairs of JAK2 or JAK2/TYK2. In vitro, GS-829845 (the primary metabolite of filgotinib) was $\approx$ tenfold less active than filgotinib, while exhibiting similar preferential inhibition of JAK1 [5]. Pharmacokinetic/pharmacodynamic modelling and simulation using data from healthy volunteers and patients with RA indicates that exposures to GS-829845 are high enough to compensate for its lower potency and the metabolite may therefore contribute to the overall pharmacodynamic effects of filgotinib [9]. In vitro studies in peripheral blood mononuclear cells and whole blood from healthy volunteers and patients with RA and confirmatory ex vivo pharmacodynamic data from healthy volunteers confirmed that filgotinib exhibits preferential inhibition of JAK1. Filgotinib inhibited JAK1-mediated signaling of IFN- $\alpha$ and IL- 6 to a similar extent to other JAK inhibitors (tofacitinib, baricitinib, upadacitinib) at doses demonstrating similar efficacy, but showed reduced inhibition of JAK-2 and JAK3-dependent pathways [10].

In patients receiving filgotinib for the treatment of moderate to severe active RA in phase 3 trials [11-13], median platelet counts remained in the normal range but decreased slightly within the first 4 weeks of treatment before becoming stable thereafter through 24 weeks' therapy [5]. Median haemoglobin values remained stable or slightly increased within the normal range during the 24 weeks, reflecting the decrease in inflammation due to disease control and preferential JAK1 inhibition [14]. Of note, filgotinib treatment is also associated with decreases in serum C-reactive protein (CRP) [as early as 2 weeks after starting treatment and maintained through 24 weeks' treatment] [5]. In pooled data from phase 2 and 3 studies, decreases in neutrophils, lymphocytes and total leucocytes occurred more frequently in the filgotinib treatment group than in other groups, and a dosedependent increase in triglycerides, total cholesterol and high-density lipoprotein levels and reduction in low-density lipoprotein levels was seen with filgotinib. LDL/HDL ratios were generally unchanged. Changes in lipid levels occurred in the first 12 weeks of treatment and then stabilized [5, 6]. 


\subsection{What is the Pharmacokinetic Profile of Filgotinib?}

Filgotinib is absorbed rapidly following oral administration, with a median peak concentration $\left(\mathrm{C}_{\max }\right)$ reached $2-3 \mathrm{~h}$ post dose (and that of its active circulating metabolite GS-829845 reached $5 \mathrm{~h}$ post dose) after multiple dosing [5]. The $\mathrm{C}_{\max }$ and exposure (area under the concentration-time curve) of filgotinib and GS-829845 are similar in healthy adults and in patients with RA, and are dose-proportional over the therapeutic dose range [5].

Filgotinib and GS-829845 reach steady-state concentrations in 2-3 days and 4 days, respectively, with negligible (filgotinib) to $\approx$ twofold (GS-829845) accumulation after oncedaily administration [5]. The administration of filgotinib with a high-fat or low-fat meal compared with a fasted state does not lead to clinically relevant differences in exposure. Human plasma protein binding is low for both filgotinib (55-59\%) and GS-829845 (39-44\%), and there is no preferential distribution of filgotinib and GS-829845 into blood cells (filgotinib bloodto-plasma ratio 0.85-1.1) [5].

Filgotinib undergoes extensive metabolism, with a low proportion of an orally administered dose recovered unchanged in the urine $(\approx 9.4 \%)$ and faeces $(\approx 4.5 \%)$ [5]. Filgotinib is metabolized primarily by carboxylesterase 2 (CES2), and to a lesser extent by CES1, both of which form GS-829845. There are no other major metabolites. Most of the dose $(\approx 87 \%)$ is eliminated in the urine ( $54 \%$ as GS-829845), and $\approx 15 \%$ is eliminated in the faeces $(\approx 8.9 \%$ as GS-829845). Filgotinib and GS-829845 have mean plasma terminal half-lives of $\approx 7 \mathrm{~h}$ and $19 \mathrm{~h}$, respectively [5].

\section{For Whom is Filgotinib Indicated?}

Filgotinib is indicated, as monotherapy or in combination with methotrexate, for the treatment of moderate to severe active RA in adults with an inadequate response or intolerance to one or more DMARDs in the EU [5]. Table 1 provides a summary of the prescribing information in the EU.

\section{What is the Clinical Efficacy of Filgotinib in RA?}

Filgotinib provides effective treatment of moderate to severe active RA in patients with an inadequate response or intolerance to DMARDs, as evidenced by improvements in clinical, patient-reported and radiographic outcomes in randomized controlled trials [11-15]. The efficacy of filgotinib in the treatment of moderate to severe active RA was initially shown in phase 2b trials (DARWIN 1 [14] and DARWIN 2 [15]), in which filgotinib as monotherapy [15] and in combination with methotrexate [14] improved the signs and symptoms of RA in patients who had an inadequate response to methotrexate [14, 15]. The efficacy of filgotinib in patients with moderate to severe active RA was subsequently evaluated in three randomized, double-blind, phase 3 trials (FINCH 1 [11], FINCH 2 [12] and FINCH 3 [13]).

The phase 3 trials enrolled patients aged $\geq 18$ years with moderate to severe active RA ( $\geq 6$ swollen joints from a count of 66 joints and $\geq 6$ tender joints from a count of 68 joints) [11-13] and an inadequate response to methotrexate [11], inadequate response to one or two conventional synthetic (cs)DMARD(s) and an inadequate response or intolerance to one or more bDMARDs [12], or limited (< 3 doses $\leq 25 \mathrm{mg}$ ) or no prior methotrexate exposure [13]. Patients with an inadequate response to methotrexate had been receiving treatment for $\geq 12$ weeks, with the most recent $\geq 4$ weeks at a stably prescribed route and dose (in the range of 7.5-25 mg/week) [11, 12]. Patients with an inadequate response to other csDMARDs had been receiving a stable dose of oral hydroxychloroquine $(\leq 400 \mathrm{mg}$ / day), chloroquine ( $\leq 250 \mathrm{mg} /$ day), sulfasalazine (1-3 g/ day) or leflunomide (10-20 mg/day) for $\geq 4$ weeks [12].

Patients were also required to have one of the following: seropositivity for rheumatoid factor (RF) and/or anti-cyclic citrullinated peptide (anti-CCP, also known as ACPA) [11, 13], $\geq 1$ joint erosion on hand/wrist or foot radiographs [11, 13] (or $\geq 3$ erosions if negative for RF and anti-CCP [11]), or serum CRP level of $\geq 4 \mathrm{mg} / \mathrm{L}[12,13]$ or $\geq 6 \mathrm{mg} / \mathrm{L}$ [11].

Key trial design data are presented as part of Table 2 . The duration of the trials was 24 weeks [12] or 52 weeks [11, 13]. Concomitant stable doses of non-steroidal anti-inflammatory drugs and/or glucocorticoids (prednisone or equivalent $\leq 10 \mathrm{mg}$ /day) were permitted [11-13]. In FINCH 3, a prior or concomitant stable dose of hydroxychloroquine was also allowed [13]; in FINCH 1, placebo recipients were rerandomized to filgotinib at week 24 [11]. The primary efficacy endpoint was the proportion of patients who achieved $\geq 20 \%$ improvement from baseline in the American College of Rheumatology criteria (ACR20) at week 12 (FINCH 1 and $2[11,12])$ or week 24 (FINCH 3[13]). Primary and key secondary endpoints were tested hierarchically [11-13]. Efficacy analyses were performed in all randomized patients who received $\geq 1$ dose of a study drug [11-13].

\subsection{Inadequate Response to Methotrexate}

Filgotinib plus background methotrexate therapy improved the signs and symptoms of RA, improved physical function and inhibited the progression of structural joint damage compared with placebo plus background methotrexate therapy in patients who had moderate to severe active RA 


\section{Table 1 Prescribing summary of filgotinib (Jyseleca ${ }^{\circledR}$ ) in the treatment of adults with rheumatoid arthritis in the EU [5]}

\section{What is the approved indication of filgotinib?}

Treatment of moderate to severe active RA in adults who have responded inadequately to, or who are intolerant to, one or more DMARDs May be used alone or in combination with methotrexate

\section{How is filgotinib available, and what is the administration regimen?}

\section{Availability}

Dosage

Limitations of use
Film-coated oral tablets of filgotinib $100 \mathrm{mg}$ or $200 \mathrm{mg}$

$200 \mathrm{mg}$ once daily, with or without food; $100 \mathrm{mg}$ once daily in some special populations (see below)

Not recommended for use in combination with other potent immunosuppressants (e.g. other JAK inhibitors, bDMARDs, azathioprine, ciclosporin, tacrolimus)

\section{What are the contraindications to the use of filgotinib?}

Pregnancy (use contraception during treatment and for $\geq 1$ week after cessation of therapy); active TB or active serious infections; hypersensitivity to the active substance or any of the excipients

\section{How should filgotinib be used in special populations?}

Pts with chronic kidney disease

Mild disease $\left(\mathrm{CL}_{\mathrm{CR}} \geq 60 \mathrm{~mL} / \mathrm{min}\right)$ : no dose adjustment required

Moderate or severe disease $\left(\mathrm{CL}_{\mathrm{CR}} 15\right.$ to $\left.<60 \mathrm{~mL} / \mathrm{min}\right): 100 \mathrm{mg}$ once daily

Kidney failure $\left(\mathrm{CL}_{\mathrm{CR}}<15 \mathrm{~mL} / \mathrm{min}\right)$ : not recommended

Pts with hepatic impairment

Mild or moderate hepatic impairment (Child-Pugh A or B): no dose adjustment required

Severe hepatic impairment (Child-Pugh C): not recommended

Elderly pts

Paediatric pts In patients aged $\geq 75$ years, start with a dosage of $100 \mathrm{mg}$ once daily

Safety and efficacy not established in pts aged $<18$ years

Do not breastfeed during treatment

What other special warnings/precautions/monitoring requirements pertain to the use of filgotinib?

Infections

Viral reactivation

Malignancy

Fertility

Haematological laboratory abnormalities

Vaccinations

Lipids

CV risk

VTE

Lactose content (excipient)

Screen pts for TB before initiating treatment; do not administer to pts with active TB; initiate standard antimycobacterial therapy in pts with latent TB before administering filgotinib

Monitor closely for infections during and after treatment; interrupt treatment if a serious infection develops, or if an infection does not respond to standard antimicrobial therapy, until it is controlled

If pt develops herpes zoster, interrupt treatment until the episode resolves

Screen for viral hepatitis and monitor for reactivation prior to and during treatment

Immunomodulatory products may $\uparrow$ the risk of malignancies (as can RA itself)

Consider the risks and benefits of filgotinib prior to initiating treatment in pts with a known malignancy

Discuss the potential risk of reduced fertility or infertility with male pts before treatment initiation

ALC $<0.5 \times 10^{9}$ cells $/ \mathrm{L}, \mathrm{ANC}<1 \times 10^{9}$ cells $/ \mathrm{L}, \mathrm{Hb}<8 \mathrm{~g} / \mathrm{dL}$ : do not initiate or continue treatment Reintroduce treatment once the parameters return above these values

Update immunisations before treatment; do not use live vaccines immediately before or during treatment

Check 12 weeks after starting filgotinib, and monitor and manage thereafter

Manage risk factors such as hyperlipidaemia and hypertension (RA pts have an $\uparrow$ risk for CV disorders)

Use with caution in pts with risk factors for VTE (e.g. older age, history of VTE, obesity, surgery)

Discontinue treatment if features of VTE occur, and promptly evaluate/treat

Do not use in pts with galactose intolerance, glucose-galactose malabsorption or total lactose deficiency

\section{What are the potential clinically relevant interactions between filgotinib and other drugs?}

CYP1A2 substrates with a narrow TI Caution recommended (in vitro studies were inconclusive, and the potential in vivo effect is unknown)

P-gp or BCRP substrates with a Caution recommended (in vitro studies were inconclusive, and in vivo inhibition cannot be excluded)

narrow TI (e.g. digoxin)

OATP1B1 and OATP1B3 substrates Caution recommended (no data; $\uparrow$ in their exposure and risk of adverse events cannot be excluded)

(e.g. valsartan, statins)

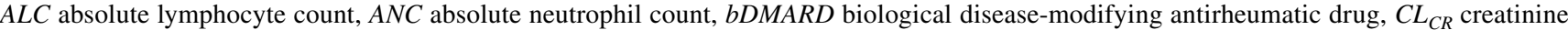
clearance, $C V$ cardiovascular, $H b$ haemoglobin, $J A K$ Janus kinase, $P$ - $g p$ P-glycoprotein, $p t s$ patients, $R A$ rheumatoid arthritis, $T B$ tuberculosis, $T I$ therapeutic index, $V T E$ venous thromboembolism (deep vein thrombosis/pulmonary embolism), $\uparrow$ increase(d) 


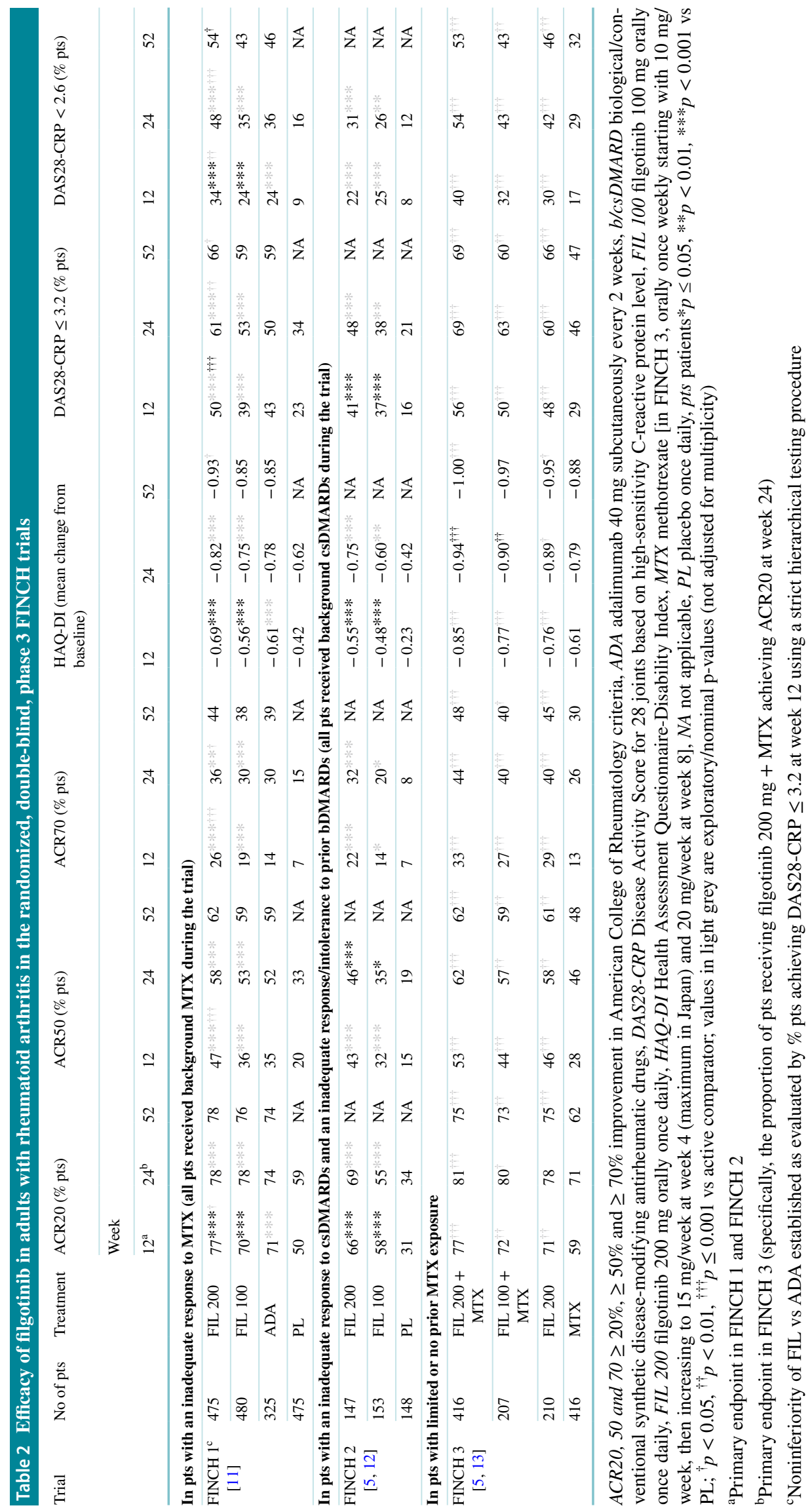


despite methotrexate therapy in the FINCH 1 trial [11]. The ACR20 response rate was significantly higher with filgotinib $200 \mathrm{mg}$ and filgotinib $100 \mathrm{mg}$ than with placebo following 12 weeks of treatment (Table 2). Significant improvements with filgotinib compared with placebo were also observed in key secondary endpoints at week 12, including change from baseline score on the Health Assessment Questionnaire-Disability Index (HAQ-DI), and proportion of patients with disease activity scores in 28 joints with CRP (DAS28CRP) $<2.6$ (considered to be disease remission) (Table 2). Of note, filgotinib $200 \mathrm{mg}$ was noninferior to adalimumab for DAS28-CRP $\leq 3.2$ (considered low disease activity) at week 12 using a strict hierarchical testing procedure (Table 2). DAS28-CRP $\leq 3.2$ and ACR50/70 response rates were also higher with filgotinib at week 12 than with placebo (nominal $p<0.001$ ) (Table 2).

Radiographic progression of joint damage, as assessed by change in van der Heijde modified total Sharp score (mTSS) from baseline to week 24 , was significantly ( $p \leq 0.001$ ) reduced with filgotinib $200 \mathrm{mg}$ and filgotinib $100 \mathrm{mg}$ versus placebo (0.13 and 0.17 vs 0.37 ) [11]. Filgotinib showed sustained efficacy through week 52 (Table 2).

\subsection{Inadequate Response to csDMARDs and Prior bDMARDs}

Filgotinib plus background csDMARD therapy improved the signs and symptoms of RA and improved physical function compared with placebo plus background csDMARD therapy in patients who had moderate to severe active RA despite ongoing csDMARD therapy and an inadequate response or intolerance to $\geq 1$ prior bDMARD in the FINCH 2 trial [12]. Of note, most patients (81.9\%) were receiving methotrexate concomitantly. The ACR 20 response rate was significantly higher with filgotinib $200 \mathrm{mg}$ and filgotinib $100 \mathrm{mg}$ than with placebo following 12 weeks of treatment (Table 2). This was independent of the number of prior bDMARDs; the proportions of patients previous treated with $\geq 3$ bDMARDs achieving ACR20 were significantly $(p<0.001)$ greater with filgotinib $200 \mathrm{mg}(70.3 \%)$ and filgotinib $100 \mathrm{mg}(58.8 \%)$ than with placebo (17.6\%) [12]. Significant improvements with filgotinib compared with placebo were also observed in key secondary endpoints at week 12 , including change from baseline in HAQ-DI, and proportions of patients achieving DAS28-CRP $\leq 3.2$ (Table 2). DAS28-CRP $<2.6$ and ACR50/70 response rates were also higher with filgotinib than with placebo at week $12(p \leq 0.05)$ (Table 2). Filgotinib showed sustained efficacy through week 24 (Table 2).

\subsection{Limited or No Prior Exposure to Methotrexate}

Filgotinib plus methotrexate therapy improved the signs and symptoms of RA, improved physical function and appeared to inhibit the progression of structural joint damage compared with methotrexate alone in patients who had moderate to severe active RA with limited or no prior methotrexate exposure in the FINCH 3 trial [13]. The ACR20 response rate was significantly higher with filgotinib $200 \mathrm{mg}$ plus methotrexate than with methotrexate alone following 24 weeks of treatment (Table 2). Significant improvements were also observed in key secondary endpoints at week 24, including the ACR20 response rate with filgotinib $100 \mathrm{mg}$ plus methotrexate therapy compared with methotrexate alone, and change from baseline in HAQ-DI and proportion of patients achieving DAS28-CRP $<2.6$ with filgotinib $200 \mathrm{mg}$ or $100 \mathrm{mg}$ plus methotrexate compared with methotrexate alone (Table 2). DAS28-CRP $\leq 3.2$ and ACR50/70 response rates were also higher with filgotinib $200 \mathrm{mg}$ or $100 \mathrm{mg}$ plus methotrexate than with methotrexate alone at week 24 (nominal $p<0.01$ ) (Table 2).

Although the ACR20 response rate was not significantly different between filgotinib $200 \mathrm{mg}$ monotherapy and methotrexate monotherapy at week 24 , the change from baseline in HAQ-DI and response rates for DAS28-CRP $\leq 3.2$, DAS28CRP $<2.6$ and ACR50/70 were higher with filgotinib $200 \mathrm{mg}$ monotherapy than with methotrexate monotherapy at week 24 (nominal $p<0.05$ ) (Table 2). Filgotinib $200 \mathrm{mg}$ monotherapy, but not filgotinib $200 \mathrm{mg}$ or $100 \mathrm{mg}$ plus methotrexate, was associated with less radiographic progression compared with methotrexate alone (least-squares mean change from baseline to week 24 in mTSS - 0.1 with filgotinib $200 \mathrm{mg}$ monotherapy vs 0.4 with methotrexate alone; nominal $p=0.006$ ) [13].

Filgotinib showed sustained efficacy (e.g. higher DAS28CRP $\leq 3.2$, DAS28-CRP $<2.6$ and ACR20/50/70 response rates with filgotinib plus methotrexate or filgotinib monotherapy than with methotrexate alone) through week 52 (Table 2). At week 52, patients receiving filgotinib $200 \mathrm{mg}$ plus methotrexate, filgotinib $100 \mathrm{mg}$ plus methotrexate or filgotinib $200 \mathrm{mg}$ monotherapy had less radiographic disease than patients receiving methotrexate alone $(0.21,0.27,0.23$ vs 0.74 ; exploratory $p<0.05$ ) [13].

\section{What is the Tolerability Profile of Filgotinib?}

Filgotinib was generally well tolerated in patients with RA in clinical trials. In pooled week 12 data from patients with RA who received filgotinib $200 \mathrm{mg}(n=777)$, filgotinib $100 \mathrm{mg}(\mathrm{n}=788)$ or placebo $(\mathrm{n}=781)$ in the placebocontrolled DARWIN 1 and 2 and FINCH 1 and 2 trials, common adverse reactions included nausea (3.5\%) [generally transient], upper respiratory tract infection (URTI; 3.3\%), urinary tract infection (UTI; $1.7 \%$ ) and dizziness $(1.2 \%)$. Neutropenia was uncommon $[5,6,16]$. Over 12 weeks, the 
incidence of infections was $17.9 \%$ with filgotinib $200 \mathrm{mg}$, $15.6 \%$ with filgotinib $100 \mathrm{mg}$ and $13.3 \%$ with placebo [16], none of which were opportunistic infections (this categorisation excluded tuberculosis) [5]. Infectious adverse drug reactions reported during 12 weeks' treatment were URTI (3.3\% with filgotinib and $1.8 \%$ with placebo), UTI (1.7\% and $0.9 \%)$, pneumonia $(0.6 \%$ and $0.4 \%)$ and herpes zoster $(0.1 \%$ and $0.3 \%)$. The incidence of serious infections was $1.0 \%$ with filgotinib $200 \mathrm{mg}, 0.9 \%$ with filgotinib $100 \mathrm{mg}$ and $0.6 \%$ with placebo $[5,16]$.

Interim results from two ongoing safety studies evaluating the effect of filgotinib on sperm parameters (MANTA-RAy in men with active RA, psoriatic arthritis, ankylosing spondylitis, or non-radiographic axial spondyloarthritis; MANTA in men with inflammatory bowel disease) indicate that at week $13,6.7 \%$ (8/120 patients) of filgotinib $200 \mathrm{mg}$ once daily recipients and $8.3 \%(10 / 120)$ of placebo recipients had $\mathrm{a} \geq 50 \%$ decline in sperm concentration [17].

The safety profile of filgotinib longer term was similar to that in the phase 2 and 3 trials $[5,18]$. No new safety concerns were identified in an integrated analysis of seven RA trials [DARWIN 1-3 and FINCH 1-4 (DARWIN 3 [18] and FINCH 4 [19] are long-term extension studies)] [20]. In a data cut including DARWIN 3 data through April 2019 and FINCH 4 data through September 2019, patients had received at least one dose of filgotinib $200 \mathrm{mg}(n=2267)$ or filgotinib $100 \mathrm{mg}(n=1640)$ for 4047.7 and 2032.9 patient-years exposure (PYE; calculated as the total exposure time in years), respectively [6]. In filgotinib $200 \mathrm{mg}$, filgotinib $100 \mathrm{mg}$, adalimumab, methotrexate and placebo recipients, the overall exposure-adjusted incidence rates (EAIRs)/100 PYEs for serious treatment-emergent adverse events (TEAEs) were 6.3, 8.2, 7.6, 7.9 and 10.3 [pneumonia was the most common across the active treatment groups $(0.4,0.5,0.7,0.8)]$ and TE death were $0.3,0.3,0.3,0$ and $0.3[6]$.

In terms of AEs of special interest (AESIs) in the integrated analysis [6], the overall EAIRs/100 PYEs in filgotinib $200 \mathrm{mg}$, filgotinib $100 \mathrm{mg}$, adalimumab, methotrexate and placebo recipients for infections were 26.5, 31.9, 44.5, 44.1 and 55.2, serious infections were 1.7, 2.5, 3.4, 2.2 and 2.3, herpes zoster virus infections were $1.8,1.1,0.7,1.1$ and 1.0, opportunistic infections were $0.1,0.2,0.7,0.6$ and 0 , venous thromboembolism were $0.2,0,0.3,0.6$ and 0.7 , nonmelanoma skin cancer were $0.2,0.1,0,0.3$ and 0 , malignancy excluding nonmelanoma skin cancer were $0.5,0.5$, 0.7, 1.1 and 1.0 and major adverse cardiovascular events (MACE) were 0.5, 0.6, 0.3, 0.6 and 1.0. Dose-dependency was not observed for the most important AESIs of MACE, malignancy or serious infections [6]. Among as-treated patients, the incidence of herpes zoster PYEs in filgotinib $200 \mathrm{mg}$, filgotinib $100 \mathrm{mg}$, adalimumab, methotrexate and placebo recipients was $3.3 \%, 1.4 \%, 0.6 \%, 1.0 \%$ and $0.4 \%$.
Most herpes zoster events were mild to moderate in severity. Multivariate analysis showed that a prior history of herpes zoster, Asian region, and age $\geq 50$ years were associated with increased risk of herpes zoster infection during filgotinib treatment [21]. Although data are limited, a higher incidence of serious infections occurred in patients aged $\geq$ 75 years (compared to those aged $<75$ years) in the filgotinib and adalimumab treatment groups; a lower starting dose of filgotinib is therefore recommended in patients aged $\geq 75$ years $[5,6]$.

\section{What is the Current Clinical Position of Filgotinib in RA?}

Filgotinib is the fourth JAK inhibitor [6] (and one of two second-generation JAK inhibitors [4]) to be approved for the treatment of RA in the EU [6]. Filgotinib, as monotherapy or in combination with methotrexate, is an effective and generally well tolerated treatment option for adults with moderate to severe active RA with an inadequate response or intolerance to $\geq 1$ DMARD, with the convenience of once-daily oral administration.

According to the 2019 European League Against Rheumatism (EULAR) recommendations for the management of RA, JAK inhibitors are recommended as an alternative to bDMARDs [2]. Methotrexate (the anchor drug in RA) is recommended as part of the initial treatment strategy (e.g. methotrexate plus short-term glucocorticoids). If there is a contraindication or early intolerance to methotrexate therapy, the (first) treatment strategy should include leflunomide or sulfasalazine. If the response is insufficient (no improvement within 3 months, or treatment target not reached by 6 months), treatment should be adjusted based on the presence or absence of poor prognostic factors. These are defined as persistently moderate or high disease activity (according to composite measures including joint counts) despite csDMARD therapy, failure of $\geq 2$ csDMARDs, high swollen joint count, presence of early erosions, high acute phase reactant levels, and presence of the autoantibodies RF and/ or ACPA (especially at high levels) [2].

In the absence of poor prognostic factors, a different csDMARD should be attempted, or a second csDMARD should be added on [2]. If poor prognostic factors are present, a bDMARD or a tsDMARD should be added to the treatment regimen [2]. Currently, the term tsDMARDs refers solely to JAK inhibitors, including tofacitinib, baricitinib, upadacitinib and filgotinib [22]. Since bDMARDs and JAK inhibitors are considered to have similar efficacy, the EULAR recommendations state that no preference can be given to either group for reasons of efficacy [2]. Instead, treatment choice may be driven by patient preference (e.g. oral route of administration) [22]. Second-generation JAK inhibitors 
such as upadacitinib and filgotinib may have a better safety profile than first-generation JAK inhibitors due to preferential inhibitory activity [1].

Recent NICE guidance [23] recommends filgotinib as an option in combination with methotrexate in adults with moderate or severe active RA (DAS28 $\geq 3.2$ ) that has responded inadequately to $\geq 2$ csDMARDs; adults with severe RA (DAS28 > 5) whose disease has responded inadequately to or who cannot have other DMARDS, including $\geq 1$ bDMARD and they cannot have rituximab; and in adults with severe RA that has responded inadequately to rituximab and $\geq 1$ bDMARD.

Filgotinib is also recommended as monotherapy in adults with moderate to severe or severe RA when methotrexate is contraindicated or cannot be tolerated [23].

Filgotinib as monotherapy or in combination with csDMARDs is effective in treating moderate to severe active RA in patients with an inadequate response or intolerance to DMARDs, providing clinically relevant disease remission or low disease activity rates, improvements in the signs and symptoms of RA and slowing of radiographic progression. Filgotinib is also as effective as adalimumab in achieving low disease activity in patients with an inadequate response to methotrextate.

Filgotinib is generally well tolerated, with the most frequently reported adverse reactions being nausea, URTI, UTI and dizziness. The frequency of serious infection with filgotinib is low and stable with longer-term exposure; the most common serious infection is pneumonia. Herpes zoster infections in the filgotinib 200 and $100 \mathrm{mg}$, active control (adalimumab, methotrexate) and placebo treatment groups in clinical trials were of comparable and low frequency. Most herpes zoster events were mild to moderate in severity; risk factors for herpes zoster infection with filgotinib include age ( $\geq 50$ years), previous herpes zoster infection and geographic location (Asia). Long-term safety evaluation in the DARWIN 3 and FINCH 4 trials is ongoing; no new safety concerns have been identified to date.

Randomized, controlled studies comparing filgotinib with other JAK inhibitors have not been conducted. Indirect comparisons using systematic reviews and meta-analyses suggest that all four approved JAK inhibitors (tofacitinib, baricitinib, upadacitinib and filgotinib) are effective options in the management of RA with inadequate responses to csDMARDs or bDMARDs, although there are some differences in their efficacy and tolerability profiles [24-27]. The risk of infection appears to be similar among approved JAK inhibitors, although herpes zoster infections may be less frequent with filgotinib [28-30]. Longer-term and real-world efficacy and safety data and cost-effectiveness analyses will be useful in further positioning filgotinib relative to other treatment options (including JAK inhibitors) in these patients.
Supplementary Information The online version contains supplementary material available at https://doi.org/10.1007/s40261-021-01055-0.

Acknowledgements The manuscript was updated from Drugs 2020;80(18):1987-97 [7], and was reviewed by: A. Becciolini, Department of Medicine, Internal Medicine and Rheumatology Unit, Azienda Ospedaliero-Universitaria di Parma, Parma, Italy; M. Harigai, Division of Rheumatology, Department of Internal Medicine, Tokyo Women's Medical University, Tokyo, Japan; $R$. Westhovens, Division of Rheumatology, University Hospitals, KU Leuven, Leuven, Belgium; $I$. Yoshii, Orthopedic Surgery, Yoshii Hospital, Shimanto City, Japan. During the peer review process, the marketing authorization holder of filgotinib was offered an opportunity to review this article. Changes resulting from comments received were made on the basis of scientific and editorial merit.

\section{Declarations}

Funding The preparation of this review was not supported by any external funding.

Authorship and Conflict of interest E. S. Kim is a contracted employee of Adis International Ltd/Springer Nature and S. J. Keam is a salaried employee of Adis International Ltd/Springer Nature, and declare no relevant conflicts of interest. All authors contributed to the review and are responsible for the article content.

Ethics approval, Consent to participate, Consent to publish, Availability of data and material, Code availability Not applicable.

Open Access This article is licensed under a Creative Commons Attribution-NonCommercial 4.0 International License, which permits any non-commercial use, sharing, adaptation, distribution and reproduction in any medium or format, as long as you give appropriate credit to the original author(s) and the source, provide a link to the Creative Commons licence, and indicate if changes were made. The images or other third party material in this article are included in the article's Creative Commons licence, unless indicated otherwise in a credit line to the material. If material is not included in the article's Creative Commons licence and your intended use is not permitted by statutory regulation or exceeds the permitted use, you will need to obtain permission directly from the copyright holder. To view a copy of this licence, visit http://creativecommons.org/licenses/by-nc/4.0/.

\section{References}

1. Angelini J, Talotta R, Roncato R, et al. JAK-inhibitors for the treatment of rheumatoid arthritis: a focus on the present and an outlook on the future. Biomolecules. 2020;10(7):1002.

2. Smolen JS, Landewe RBM, Bijlsma JWJ, et al. EULAR recommendations for the management of rheumatoid arthritis with synthetic and biological disease-modifying antirheumatic drugs: 2019 update. Ann Rheum Dis. 2020;79(6):685-99.

3. Ciobanu DA, Poenariu IS, Crîngus L-I, et al. JAK/STAT pathway in pathology of rheumatoid arthritis. Exp Ther Med. 2020;20(4):3498-503.

4. Banerjee S, Biehl A, Gadina M, et al. JAK-STAT signaling as a target for inflammatory and autoimmune diseases: current and future prospects. Drugs. 2017;77(5):521-46. 
5. European Medicines Agency. Filgotinib (Jyseleca): EU summary of product characteristics. 2020. https://www.ema.europa.eu/. Accessed 18 May 2021.

6. European Medicines Agency. Jyselca: CHMP assessment report. 2020. https://www.ema.europa.eu/. Accessed 18 May 2021.

7. Dhillon S, Keam SJ. Filgotinib: first approval. Drugs. 2020;80(18):1987-97.

8. Van Rompaey L, Galien R, van der Aar EM, et al. Preclinical characterization of GLPG0634, a selective inhibitor of JAK1, for the treatment of inflammatory diseases. J Immunol. 2013;191(7):3568-77.

9. Namour F, Diderichsen PM, Cox E, et al. Pharmacokinetics and pharmacokinetic/pharmacodynamic modeling of filgotinib (GLPG0634), a selective JAK1 inhibitor, in support of phase IIB dose selection. Clin Pharmacokinet. 2015;54(8):859-74.

10. Traves PG, Murray B, Campigotto F, et al. JAK selectivity and the implications for clinical inhibition of pharmacodynamic cytokine signalling by filgotinib, upadacitinib, tofacitinib and baricitinib. Ann Rheum Dis. 2021:80(7):865-75.

11. Combe B, Kivitz A, Tanaka Y, et al. Filgotinib versus placebo or adalimumab in patients with rheumatoid arthritis and inadequate response to methotrexate: a phase III randomised clinical trial. Ann Rheum Dis. 2021:80(7):848-58.

12. Genovese MC, Kalunian K, Gottenberg JE, et al. Effect of filgotinib vs placebo on clinical response in patients with moderate to severe rheumatoid arthritis refractory to disease-modifying antirheumatic drug therapy: the FINCH 2 randomized clinical trial. JAMA. 2019;322(4):315-25.

13. Westhovens R, Rigby WFC, van der Heijde D, et al. Filgotinib in combination with methotrexate or as monotherapy versus methotrexate monotherapy in patients with active rheumatoid arthritis and limited or no prior exposure to methotrexate: the phase 3, randomised controlled FINCH 3 trial. Ann Rheum Dis. 2021;80(6):727-38.

14. Westhovens R, Taylor PC, Alten R, et al. Filgotinib (GLPG0634/ GS-6034), an oral JAK1 selective inhibitor, is effective in combination with methotrexate (MTX) in patients with active rheumatoid arthritis and insufficient response to MTX: results from a randomised, dose-finding study (DARWIN 1). Ann Rheum Dis. 2017;76(6):998-1008.

15. Kavanaugh A, Kremer J, Ponce L, et al. Filgotinib (GLPG0634/ GS-6034), an oral selective JAK1 inhibitor, is effective as monotherapy in patients with active rheumatoid arthritis: results from a randomised, dose-finding study (DARWIN 2). Ann Rheum Dis. 2017;76(6):1009-19.

16. Galloway J, Buch MH, Yamaoka K, et al. Infections and serious infections in the filgotinib rheumatoid arthritis program [abstract no. OP0126]. In: EULAR 2021 Congress. 2021.

17. Galapagos NV. Galapagos reports primary endpoint for the ongoing filgotinib MANTA and MANTA-RAy safety studies [media release]. 4 Mar 2021. https://www.glpg.com/.
18. Kavanaugh A, Westhovens RR, Winthrop KL, et al. Safety and efficacy of filgotinib: up to 4-year results from an open-label extension study of phase 2 rheumatoid arthritis programs. J Rheumatol. 2021. https://doi.org/10.3899/jrheum.201183.

19. Gilead Sciences. Long term extension study to assess the safety and efficacy of filgotinib in adults with rheumatoid arthritis (FINCH 4). 2021. https://www.clinicaltrials.gov/ct2/show/ NCT03025308. Accessed 15 May 2021.

20. Genovese MC, Winthrop K, Tanaka Y, et al. Integrated safety analysis of filgotinib treatment for rheumatoid arthritis from 7 clinical trials [abstract no. THU0202]. Ann Rheum Dis. 2020;79(Suppl. 1):324-5.

21. Winthrop K, Buch MH, Curtis JR, et al. Herpes zoster in the filgotinib rheumatoid arthritis program [abstract no. POS0092]. In: EULAR 2021 Congress. 2021.

22. Richez C, Truchetet ME. What value do JAK inhibitors have in treating rheumatoid arthritis? Expert Opin Pharmacother. 2020;21(15):1789-92.

23. National Institute for Health and Care Excellence. Filgotinib for treating moderate to severe rheumatoid arthritis. 2021. https:// www.nice.org.uk/guidance/ta676. Accessed 6 Apr 2021.

24. Lee YH, Song GG. Comparative efficacy and safety of tofacitinib, baricitinib, upadacitinib, and filgotinib in active rheumatoid arthritis refractory to biologic disease-modifying antirheumatic drugs. Z Rheumatol. 2021;80(4):379-92.

25. Lee YH, Song GG. Comparison of the efficacy and safety of tofacitinib and filgotinib in patients with active rheumatoid arthritis: a Bayesian network meta-analysis of randomized controlled trials. Z Rheumatol. 2020;79(6):590-603.

26. Jacob I, Beresford L, Butler K, et al. Comparative efficacy of filgotinib versus alternative treatments for rheumatoid arthritis in patients with an inadequate response to biologic diseasemodifying antirheumatic drugs: a systematic review and network meta-analysis [abstract no. PMS5]. Value Health. 2020;23(Suppl. 2):S592-3

27. Jacob I, Beresford-Hulme L, Butler K, et al. Comparative efficacy of filgotinib versus alternative treatments for rheumatoid arthritis in patients with an inadequate response to methotrexate: a systematic review and network meta-analysis [abstract no. PMS4]. Value Health. 2020;23(Suppl. 2):S592.

28. Alves C, Penedones A, Mendes D, et al. The risk of infections associated with JAK inhibitors in rheumatoid arthritis: a systematic review and network meta-analysis. J Clin Rheumatol. 2021. https://doi.org/10.1097/RHU.0000000000001749.

29. Clarke B, Yates M, Adas M, et al. The safety of JAK-1 inhibitors. Rheumatology (Oxford). 2021;60(Suppl 2):ii24-30.

30. Harigai M, Honda S. Selectivity of Janus kinase inhibitors in rheumatoid arthritis and other immune-mediated inflammatory diseases: is expectation the root of all headache? Drugs. 2020;80(12):1183-201. 